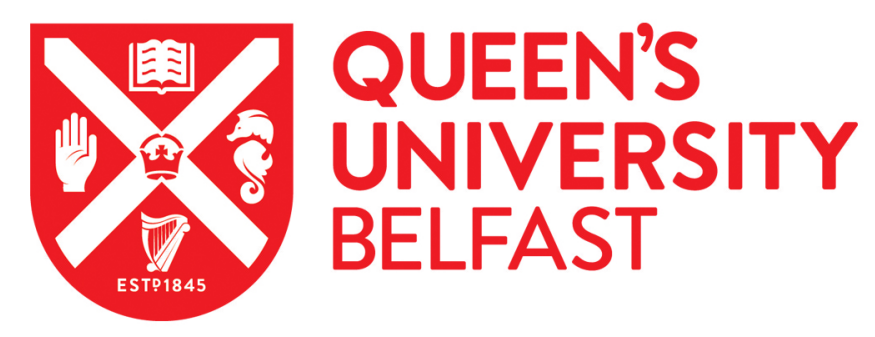

\title{
A plasmonic biosensor array exploiting plasmon coupling between gold nanorods and spheres for domoic acid detection via two methods
}

Neils, J. L. D., Salvador, J. P., Marco, M. P., Elliott, C. T., \& Campbell, K. (2021). A plasmonic biosensor array exploiting plasmon coupling between gold nanorods and spheres for domoic acid detection via two methods. SPECTROCHIMICA ACTA PART A-MOLECULAR AND BIOMOLECULAR SPECTROSCOPY. https://doi.org/10.1016/j.saa.2021.119473

Published in:

SPECTROCHIMICA ACTA PART A-MOLECULAR AND BIOMOLECULAR SPECTROSCOPY

Document Version:

Peer reviewed version

Queen's University Belfast - Research Portal:

Link to publication record in Queen's University Belfast Research Portal

Publisher rights

Copyright 2021 Elsevier.

This manuscript is distributed under a Creative Commons Attribution-NonCommercial-NoDerivs License

(https://creativecommons.org/licenses/by-nc-nd/4.0/), which permits distribution and reproduction for non-commercial purposes, provided the author and source are cited.

\section{General rights}

Copyright for the publications made accessible via the Queen's University Belfast Research Portal is retained by the author(s) and / or other copyright owners and it is a condition of accessing these publications that users recognise and abide by the legal requirements associated with these rights.

Take down policy

The Research Portal is Queen's institutional repository that provides access to Queen's research output. Every effort has been made to ensure that content in the Research Portal does not infringe any person's rights, or applicable UK laws. If you discover content in the Research Portal that you believe breaches copyright or violates any law, please contact openaccess@qub.ac.uk. 


\section{A plasmonic biosensor array exploiting plasmon coupling between gold nanorods and spheres for domoic acid detection via two methods}

Joost L.D. Nelis ${ }^{1 *}$, J. Pablo Salvador ${ }^{2-3}$, M. Pilar Marco ${ }^{3-2}$, Christopher T. Elliott $^{1}$ \& Katrina Campbell ${ }^{1}$

1. Institute for Global Food Security, School of Biological Sciences, Queen's University, 19 Chlorine Gardens, Belfast, UK BT9 5DL

2. CIBER de Bioingeniería, Biomateriales y Nanomedicina (CIBER-BBN), Jordi Girona 18-26, 08034 Barcelona, Spain

3. Nanobiotechnology for Diagnostics (Nb4D), Institute for Advanced Chemistry of Catalonia (IQAC) of the Spanish Council for Scientific Research (CSIC), Jordi Girona 18-26, 08034 Barcelona, Spain

*Corresponding Author:

J.L.D. Nelis

Email: J.Nelis@qub.ac.uk

Tel: $+44(0) 7305143761$ 


\section{Abstract}

An immunoassay was developed that utilized plasmonic coupling between immobilised gold nanorods and colloid gold nanospheres to detect the marine toxin domoic acid (DA). The aspect ratio of the nanorods was optimised and the effects of variation in acidity, silver to gold ratio, cetyltrimethylammonium bromide (CTAB) concentration and seed addition in the growth solution on the yield, size variance and LSPR peak position was investigated. Excellent nanorods (size variation $<15 \%$; aspect ratio 3.5-5; yield 0.26-0.35 $\mathrm{nM} \mathrm{mL}^{-1}$ ) were obtained for the LSPR range $785-867 \mathrm{~nm}$ using strong acidic conditions (12 $\mu \mathrm{L} \mathrm{HCl}(37 \%))$, silver to gold ratio of $1: 5,0.05-0.1 \mathrm{M} \mathrm{CTAB}$ and $20-30 \mu \mathrm{L}$ seed addition to $10 \mathrm{~mL}$ of growth solution. One set of nanorods ( $54.9 \times 15.7 \mathrm{~nm}$; LSPR $785 \mathrm{~nm}$ ) were immobilised onto a silica support and bio-functionalised with DA hapten. Colloid nanospheres ( $15 \mathrm{~nm}$; LSPR 519nm) were bio-functionalised with an anti-domoic-acid monoclonal antibody. The functionalised nanoparticles were used to detect DA by plasmon coupling by quantifying the average LSPR shift of individual plasmon couples with hyperspectral imaging or quantifying the pixels count caused by the particle aggregation visible under darkfield microscopy. The first method led to a LSPR blue-shift of $\sim 55 \mathrm{~nm}$ caused by the immunoreaction. The second, simpler method, enabled very clear qualitative detection $(\mathrm{p}<0.0005)$ of domoic acid when $10 \mu \mathrm{M}$ domoic acid was added. Both methods show potential though the novelty and simplicity of the second platform allowing rapid ( $\sim 30$ minutes) detection with high-throughput possibilities using a simple set-up is of most interest.

Keywords: plasmonic coupling, hyperspectral imaging, darkfield microscopy, marine toxin, biosensor, nanorod synthesis 


\section{Introduction}

Biosensing through localised surface plasmon resonance (LSPR) is a powerful tool for rapid analyses that can be used for label free quantification, in real time [1]. However, the sensitivity of such systems may be limited due to small changes in the refractive index (RI). To enhance the sensitivity, anisotropic nanoparticles, with better RI sensitivity, can be used instead of isotropic particles [2],[3]. Another option is signal enhancement through plasmon coupling since bringing two plasmonically active species together often effects the LSPR frequency stronger as a RI change [4]. Indeed, plasmonic coupling of nanospheres has been shown to lead to a three-fold bulk RI sensitivity increase compared to single, well separated nanospheres [5]. Moreover, particle size and combination may influence the plasmonic coupling with larger anisotropic particles producing better RI sensitivity and smaller nanospheres enabling close proximity cluster formations and stronger coupling effect making such a combination particularly attractive [6]. Indeed, this strategy was successfully used to improve sensitivity by the van Duyne group. They showed a four-fold amplification of the shift when small $(\sim 20 \mathrm{~nm})$ gold nanoparticle labelled antibody was used instead of comparable concentrations of antibody in a proof of principle study using biotin/anti-biotin and silver nanoprisms [7]. In another study, it was shown that gold nanorods were an excellent nanomaterial to create biosensing chips since the aspect ratio (AR) and LSPR frequency of the particles can be controlled during synthesis to increase the RI sensitivity [8]. The RI sensitivity of nanorods is directly linked to the aspect ratio. A higher AR increases RI sensitivity but red shifts the longitudinal LSPR (L-LSPR) peak. Unfortunately, nanorods with high ARs are unfit to use with lowcost instrumentation using CMOS cameras or even CCD cameras since the quantum efficiency of both cameras is drastically reduced at the L-LSPR peak $(900-1000 \mathrm{~nm})$ of such nanorods [9]. Moreover, the full-width-half-maximum often widens for nanoparticles with highly red or blue shifted LSPR peaks leading to a possible trade-off between LSPR tuning towards the blue or red limits and sensitivity. This problem was elegantly illustrated by Becker et al.,. by showing that the quality factor, which describes the number of oscillations that occur until the oscillation is damped, has its 
highest maximum at an AR around 3.3 [10]. Thus, an AR of about 3-4 (which corresponds with an LSPR peak between 700 and $800 \mathrm{~nm}$ ) can be considered as the ideal working range for an LSPR based spectroscopic sensor.

Nanorod synthesis is often performed using a cost efficient bottom-up seed mediated growth method where the amount of added seed in the second growth can be varied to tune the AR [11], [12]. Various alternative bottom-up pathways for LSPR tuning have been investigated such as varying the amount of added silver (which affects availability of various facets of the nuclei for reduction) or hydrochloric acid (effecting the redox potential of the reducing agent and under-potential deposition of silver ions on the $\mathrm{Au}^{0}$ surface) or cetyltrimethylammonium bromide (CTAB; effecting reduction kinetics) [13][18]. However, the efficiency of these pathways to obtain homogeneous rods with optimised synthesis yields and the effects of combining these methods is rarely investigated even though it has been observed that the effects these individual parameter have on the AR are highly inter-dependent [19]. Another way to exploit plasmonic coupling is the detection of particle aggregation with darkfield microscopy (DFM) by measuring Rayleigh scattering intensity. This technique was recently used for the detection of glutathione-cadmium(II) complexes using unmodified gold nanospheres [20] and bioconjugated gold and silver nanospheres for the detection of cancer biomarkers in serum [21]. The method is interesting since it does not require the reconstruction of spectroscopic spectra of the individual particles making data analyses more straightforward. This being said, it may also be interesting to look at the pixel count as a more direct measurement of particle aggregation instead. To the best of the authors' knowledge that alternative has not been investigated to date.

In this study, the effects of plasmonic coupling on LSPR shifts of nanoparticles with various anisotropy has been tested for the detection of domoic acid (DA) using a competitive immunoassay setup. This marine toxin was selected as a model compound for which rapid screening tests are urgently needed due to the increased risk for food safety in the rapidly growing aquaculture industry caused by a globally increased marine toxin occurrence triggered by climate change and eutrophication [22]-[25]. 
Gold nanorods were synthesized and tuned for the L-LSPR to fall within 700-850 nm using seed, silver to gold ratio, CTAB concentration and acidity mediated approaches individually as well as in combination. Combining these mechanisms greatly improved the yield and homogeneity of the nanorods at given ARs. Nanorods (LSPR $784 \mathrm{~nm}$; AR 3.5) were then immobilised on a silica support and functionalised with a previously developed DA-protein conjugate (NR-DA-BSA) [26]. Gold nanospheres were synthesized and functionalised with anti-DA monoclonal antibody (AntiDA-NP) [27]. A wellknown synthesis protocol was followed for nanosphere synthesis of relatively small ( $20 \mathrm{~nm})$ particles to ensure maximum cluster formation and plasmon coupling potential while maintaining ease of fabrication. That said, the performance of alternative nanosphere sizes may influence plasmon coupling and this may be investigated in an additional study. Two methods were utilized to investigate if plasmonic coupling of these particles could be used for DA detection using a microarray set-up. The first technique used spectroscopic reconstruction of the average extinction spectra of the particle aggregates. The second technique used pixel extraction to detect the particle aggregation. The complete process of nanoparticle immobilisation, bioconjugation of immobilised nanorods with DAconjugate, bioconjugation of colloid nanospheres with anti-DA monoclonal antibody and DA detection by both methods is shown in fig. 1 .

\section{Material and methods}

\subsection{Materials, equipment and software}

Sinapinic acid, acetonitrile, formic acid, anhydrous sodium borate, sodium carbonate decahydrate, $\mathrm{CaSO}_{4}$, domoic acid, boric acid, bovine serum albumin (BSA), TWEEN ${ }^{\circledR} 20$, Phosphate buffer saline tablets, $\mathrm{AgNO}_{3}, \mathrm{HAuCl}_{4}$, L-Ascorbic acid, ethanol, sodium citrate, $\mathrm{HNO}_{3}(70 \%), \mathrm{HCl}(37 \%), \mathrm{NaOH}, \mathrm{H}_{2} \mathrm{SO}_{4}$, $\mathrm{H}_{2} \mathrm{O}_{2}$ (30\%), N,N'-Dicyclohexylcarbodiimide (DCC), 1-Ethyl-3-(3-dimethylaminopropyl)carbodiimide (EDC), N-hydroxysuccinimide (NHS), 3-glycidoxypropyltrimethoxysilane (GPTMS), dimethylformamide (DMF), glass slides ( $75 \times 25 \mathrm{~mm}$; thickness 0.96 to $1.06 \mathrm{~mm}$ ) and polyclonal goat anti-mouse antibody conjugated to tetramethylrodamine isothiocyanate (anti-IgG-TRITC) were purchased from Sigma Aldrich. HRP conjugated goat anti-mouse IgG (HRP-pAB) was purchashed from Dako, Agilent. O-(2Page $\mid 5$ 
Mercaptoethyl)-O'-(2-carboxyethy)heptaethyleneglycol (0.46 kDa PEGacid; MW 458.6) and 2,5,8,11,14,17,20-heptaoxadocosane-22-thiol (mPEG; MW 356.5) were purchased from Polypure. SHPEG-NH 2 (1 kD PEGamine; MW 1kD) and SH-PEG-COOH (5 kD PEGacid; MW 5 kD) were obtained from Lysan Bio. AntiDA-mAb was produced in-house and previously characterized [27]. A Cary 60 UV-Vis spectrometer (Agilent) was used for spectroscopic measurements. The Transmission Electron Microscope (TEM) was a Jeol JEM-1400 Plus equipped with JEOL 'Ruby' 8MP Bottom Mounted CCD Digital Camera (JEOL, U.K.) with a0.32 nm ultimate resolution. Samples were prepared on a formvar carbon mesh (Agar scientific) by carefully pipetting $4 \mu \mathrm{L}$ of colloid nanoparticles (at an optical density of approximately 1.5$)$ onto the mesh. An accelerating voltage of $120 \mathrm{kV}$ was used and various magnifications were applied to visualize the particles. A compartmentalised holder (Arrayit, $8 \times 3$ slots) was used to functionalise the glass slides with nanoparticles. A fluorescence scanner (InnoScan710A; Innopsys) was used for fluorescence measurements. A BX43 microscope (Olympus, Germany) fitted with an oblique angle lighting system sourced from a fibre optic cable directed halogen light source $(100 \mathrm{~W})$ that was directed into an adjustable immersion-oil condenser (numerical aperture $=1.4-1.2$ ) was used to create darkfield images. The light scattered by the particles was collected by a long working distance objective lens of $40 \mathrm{X}$ or $4 \mathrm{X}$ depending on the measurement and images were taken using a CCD camera (Q-imaging). Hyperspectral images were taken using the Cytoviva imaging system (v4.8). Individual particle (or particle cluster) spectra were collected using a particle filter available in the software and exported to be further treated using graphpad v6.1. Statistical and regression analyses were performed in Graphpad. All nanoparticle measurements were performed in ImageJ V1.52a.

\subsection{Nanoparticle synthesis}

Piranha and aqua regia solution were used consecutively to remove organic and inorganic residues on the glassware. Nanospheres were synthesized following the Turkevich method [28]. This easy-to-use method was chosen to ensure a broad application potential. Briefly, $500 \mu \mathrm{L}$ of $100 \mathrm{mM} \mathrm{HAuCl}_{4}$ was added to $194.5 \mathrm{~mL} \mathrm{MQ}$ and the solution was brought to boil. Sodium citrate $(5 \mathrm{~mL} ; 1 \%(\mathrm{w} / \mathrm{v}))$, was 
added at boiling point and the solution was left boiling for 30 minutes then cooled. Transmission electron microscopy (TEM) and UV-Vis characterisation of these particles was reported previously and allowed for size estimations of $15.1 \pm 1.7 \mathrm{~nm}$ and $13.9 \pm 2.9 \mathrm{~nm}$ respectively [26], [29]. Nanosphere concentration was estimated following [30]. For nanorod synthesis gold nuclei (seeds) are produced by adding $\mathrm{CTAB}(2 \mathrm{~mL} ; 0.2 \mathrm{M})$ to $\mathrm{HAuCl}_{4}(2 \mathrm{~mL} ; 0.5 \mathrm{mM})$ with vigorous stirring and reducing the gold salt with $240 \mu \mathrm{L}$ of freshly prepared ice-cold $\mathrm{NaBH}_{4}(0.01 \mathrm{M})$ [11], [12]. The stirred solution turned brownish yellow, indicating nucleation. The mixture was left for 30 minutes at room temperature to assure maturation while preparing the growth solution. For the growth solution various amounts of $\mathrm{AgNO}_{3}$ (gold:silver molar ratios of 1:5, 1:10, 1:25 and 1:50) were added to $5 \mathrm{~mL} \mathrm{CTAB}(0.2$ or $0.1 \mathrm{M}$ ) and left undisturbed for 15 minutes after which $5 \mathrm{~mL}$ of $0.5 \mathrm{mM} \mathrm{HAuCl}_{4}$ and various amounts of $\mathrm{HCl}$ (37\%; 0-12 $\mu \mathrm{L}$ ) or $\mathrm{NaOH}(5 \mathrm{M} ; 2$ and $4 \mu \mathrm{L}$ ) were added under slow stirring. Next $75 \mu$ l of ascorbic acid (79 mM) was added to the solution and shaken vigorously upon which the solution turned colourless. Various amounts of the seed solution $(10-150 \mu \mathrm{L})$ were then added. The mixture was shaken (30 seconds) and left overnight at $28{ }^{\circ} \mathrm{C}$. Nanorod concentrations were estimated using experimentally determined epsilon values for nanorods with comparable L-LSPR peaks and aspect ratios [31].

\subsection{Nanoparticle SAM formation}

The steps described here were for $2 \mathrm{~mL}$ of synthesised nanoparticles. For nanospheres, excess sodium citrate was removed by centrifuging at $11.400 \mathrm{RCF}$ for 30 minutes and resuspending in $2 \mathrm{~mL} \mathrm{MQ}$ (twice). Nanorods were centrifuge-d at 11.400 RCF for 15 minutes and resuspended in $2 \mathrm{~mL}$ MQ then centrifuged at $8000 \mathrm{RCF}$ for 12 minutes and resuspended in $2 \mathrm{~mL}$ of MQ. In the third centrifugation, 3000 RCF for 15 minutes centrifugation was performed and the pellets were collected. The supernatant was centrifuged again at 5500 RPM for 15 minutes and the pellet was added to the previous collected pellet. Nanorod and nanosphere pellets were resuspended in $5 \mathrm{~mL} \mathrm{MQ}$ for longterm storage at $4{ }^{\circ} \mathrm{C}$. Nanorod centrifugations were performed at $30^{\circ} \mathrm{C}$ to avoid CTAB precipitation. Nanospheres were centrifuged at $4{ }^{\circ} \mathrm{C}$. Various heterobifunctional polyethylene glycol (PEG) molecules were used to stabilise the particles. For nanospheres a mixture of $0.46 \mathrm{kD}$ PEGacid:mPEG 
in a 1:3(vol:vol) ratio was used. This mixture was used since it was found that a lower density of the functional group leads to higher conjugation success rate (likely due to better charge distribution on the particles) [32]. For nanorods 0.46 kDa PEGacid:mPEG in a 1:3(vol:vol) ratio, 5 kDa PEGacid, 5 kDa PEGacid mixed with mPEG (1:3(vol:vol) ratio), 1 kDa PEGamine and 1 kDa PEGamine and mPEG in a 1:3 ratio were tried. PEG concentration was $10 \mu \mathrm{M}$ for nanorods (since CTAB can be difficult to replace) and $1 \mu \mathrm{M}$ for nanospheres. The particles were mixed with the PEGs after clearing the Eppendorfs with a nitrogen flow to prevent oxidation of the thiol groups. The mixtures were slowly agitated for 4 hours after which stability was accessed visually. Nanoparticles were centrifuged after SAM formation to remove access reagent at the previously detailed speeds and temperatures.

\subsection{Particle immobilisation}

The functionalization of glass slides was performed according the procedure describer by Tort et al. [33] with slight modifications. Precleaned glass slides were thoroughly cleaned with water and soap, immersed in piranha solution (30 minutes) and washed 6 times with MQ. They were then immersed in a $10 \% \mathrm{NaOH}$ solution (30 minutes) and washed thrice with $\mathrm{MQ}$ and ethanol. The clean slides were dried in $\mathrm{N}_{2}$, treated with $300 \mu$ l GPTMS (30 minutes), washed and sonicated for 20 minutes in ethanol and finally dried under $\mathrm{N}_{2}$ and stored under vacuum. For nanorod immobilisation, the slides were fitted

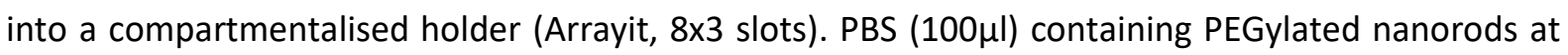
different dilutions were added to the compartments for 30 minutes followed by a thorough wash in $\mathrm{MQ}$ and drying under $\mathrm{N}_{2}$.

\subsection{Bioconjugate preparation}

A previously characterised BSA-DA bioconjugate was used and synthesis is detailed in the supplementary material [26].

\subsection{Bioconjugation}

For PEGylated nanospheres the carboxylic group was activated by adding $2.5 \mathrm{mM}$ EDC and $1.25 \mathrm{mM}$ NHS in phosphate buffer (50 mM; pH 7.5) containing $8 \% \mathrm{NaCl}$ (PBS) to the pellet up to a volume of 2 
$\mathrm{mL}$, following Fernández et al., [34]. The mixture was agitated for 30 minutes then centrifuged (11.400 RCF; 30 minutes) and the supernatant discarded. The pellet was brought up to $2 \mathrm{~mL}$ with a borate buffer ( $50 \mathrm{mM}$ boric acid; $5.6 \mathrm{mM}$ sodium borate; $\mathrm{pH} 8.7$ ) and $8 \mu \mathrm{g}$ of antibody (AntiDA-mAb or aspecific monoclonal antibody directed against neamine (Aspec-mAb)) was added. This mixture was agitated (4 hrs), pelleted (11.400 RCF centrifugation; 30 minutes) and resuspended in $2 \mathrm{~mL}$ PBS and stored at $4{ }^{\circ} \mathrm{C}$. The used antibody amount was chosen since it was calculated that $\sim 8 \mu \mathrm{g}$ antibody is needed for the functionalisation of nanospheres with a monolayer (using nanosphere concentration estimates and a diameter of an average IgG molecule of 13.7 by $8.4 \mathrm{~nm}$ ) [30], [35].

PEGylated immobilised nanorods were functionalised with BSA-DA via a one-step EDC/NHS mechanism. Briefly, $100 \mu \mathrm{L}$ of $2.5 \mathrm{mM}$ EDC and $1.25 \mathrm{mM}$ NHS in PBS containing $0.1 \mu \mathrm{g}$ BSA-DA was added to each slot in the holder for 2 Hrs after which the slide was washed with PBS containing $0.05 \%$ tween (PBST). The used amount of DA-BSA per well was chosen as it was calculated that would create a monolayer (using particle surface/concentration calculations [31] and the assumption that the diameter of a BSA-DA molecule is about 3nm (Bionumbers ID: 103736)).

\subsection{Fluorescence assays}

Immobilised nanorods (functionalised with BSA-DA or not) were exposed to PBS or a AntiDA-mAb in PBS (100 $\left.\mu \mathrm{L}, 10 \mu \mathrm{g} \cdot \mathrm{mL}^{-1}\right)$ for 30 minutes, washed with PBST and incubated with anti-IgG-TRITC (100 $\mu \mathrm{L}, 5 \mu \mathrm{g} \cdot \mathrm{mL}^{-1}$ ) in PBS for 30 minutes. Slides were then washed with PBST and dried under $\mathrm{N}_{2}$. The acquired signal was quantified (50 random points per sample, 2 samples per condition) using the fluorescence scanner at $576 \mathrm{~nm}$. The noise produced by the unfunctionalised particles was quantified as well and used to determine the $\mathrm{S} / \mathrm{N}$ ratio.

\subsection{Domoic acid detection methods}

In the first method DFM images were taken of individual spots (with a 40X objective; $n=3$ ) of NR-DABSA before and after incubation (30 minutes) with $100 \mu \mathrm{L}$ of a 10X dilution of AntiDA-NP and washing three times in PBST. A darkfield microscope and hyperspectral imaging software (Cytoviva) was used to visualise the particle extinctions, select regions of interest (ROIs) within spots and reconstruct the 
spectra of the particle couples/aggregate extinctions in the ROIs using identical filter criteria before and after the immunoreaction [36]. The LSPR shift was determined as the difference between the average spectra before and after the immunoreaction.

For the second method, spots of BSA-DA-NRs were incubated (30 minutes) with $100 \mu \mathrm{L}$ of 1:10 dilutions of PEGylated nanospheres (PEG-NPs), AntiDA-NPs s, Aspec-mAb-NPs or BSA-DA-NPs with 10 $\mu \mathrm{M}$ DA addition and washed three times with PBST. DFM and hyperspectral images of individual spots (with a $4 \mathrm{X}$ objective; $\mathrm{n}=3$ ) with BSA-DA-NRs were taken before and after these incubations and used to select ROls in Cytoviva at an identical filter threshold. The obtained total pixel counts per spot before and after immunoreactions were compared. At least 100 particles or particle aggregates per spot were detected for each measurement for both detection methods.

\section{Results and discussion}

\subsection{Individual tuning mechanisms}

\subsubsection{Seed mediated and silver mediated approach.}

Nanorods were tuned by varying the amount of seed solution (10-150 $\mu \mathrm{L})$ added to the growth solutions containing 1:5, 1:10, $1: 25$ and 1:50 of $\mathrm{AgNO}_{3}: \mathrm{HAuCl}_{4}$ molar ratios, $0.05 \mathrm{M} \mathrm{CTAB}$ and $12 \mu \mathrm{l}$ HCL (37\%) (fig. 2a). The 1:5 and 1:10 ratio's allowed to tune the LSPR peaks over a similar absolute range using 10-60 $\mu \mathrm{l}$ seed addition, albeit that nanorods synthesised at a 1:10 silver: gold ratio were more blue shifted, had lower OD values and a slightly larger full-width-half-maximum. Increasing seed addition to $150 \mu \mathrm{l}$ (fig. 2a; broken lines) did not increase LSPR red shift and caused a decrease in the maximum OD values. Thus, utilizing seed addition for LSPR tuning was limited to a maximum of $60 \mu \mathrm{l}$ in all further experiments. For lower silver concentrations (ratios 1:25 and 1:50) poor synthesis was achieved with poor maximum OD values and low L-LSPR to transversal LSPR (T-LSPR) ratios. Combining seed mediated tuning with varying the silver to gold ratios between 1:5 and 1:10 maximum made it possible to tune the L-LSPR between $\sim 780$ and $900 \mathrm{~nm}$. 


\subsection{2 $\mathrm{HCl}$ mediated tuning}

The addition of $\mathrm{HCl}(37 \%)$ to the growth solution was varied $(12-0 \mu \mathrm{l})$. Finally, the $\mathrm{pH}$ of two more solutions was altered by adding 2 and $4 \mu \mathrm{I} \mathrm{NaOH}(5 \mathrm{M})$ to the growth solution. The Ag to Au ratio was 1:5. CTAB concentration was $0.05 \mathrm{M}$ and $60 \mu \mathrm{l}$ of seed was added to the growth solution. Normalised UV-Vis spectra are shown in fig. 2b. Fig. 2c shows the L-LSPR peak position and absorbance at that value. The L-LSPR peak can be tuned over $\sim 900-650 \mathrm{~nm}$ in this manner (fig. $2 \mathrm{~b}$ ). However, reducing acidity negatively effects the yield (fig. 2 c) and L-LSPR: T-LSPR ratio indicating higher levels of impurities. This may be due to faster, less controlled $\mathrm{AuCl}_{4}^{-}$reduction at the $\mathrm{Au}^{0}$ surface at lower acidity. In order to obtain a better yield of nanorods that feature a L-LSPR peak around $700 \mathrm{~nm}$ it may be interesting to combine $\mathrm{pH}$ mediated tuning with other methods that slow down reaction kinetics.

\subsubsection{CTAB concentration}

Nanorods were synthesised using either 20 or $60 \mu$ seeds in a growth pot with a CTAB concentration of $0.05 \mathrm{M}$ or $0.1 \mathrm{M}$ and $1: 5$ gold to silver ratio (fig. $2 \mathrm{~d}$ ). The nanorods synthesized at $0.1 \mathrm{M} \mathrm{CTAB}$ were red shifted from the nanorods synthesized at $0.05 \mathrm{M} \mathrm{CTAB}$ for both seed additions, indicating better anisotropic growth at 0.1M CTAB. The full-width-half-maximum of the curves are quite similar, be it slightly reduced in the $0.1 \mathrm{M}$ CTAB synthesis, with $184 \mathrm{~nm}$ at $20 \mu \mathrm{l}$ seed addition and $171 \mathrm{~nm}$ at $60 \mu \mathrm{l}$ seed addition for the $0.05 \mathrm{M} \mathrm{CTAB}$ synthesis and 151 and $173 \mathrm{~nm}$ at $20 \mu \mathrm{l}$ and $60 \mu \mathrm{l}$ seed addition for the $0.1 \mathrm{M}$ CTAB synthesis. However, when $60 \mu \mathrm{l}$ seed addition and 0.05M CTAB was used the L-LSPR:TLSPR ratio was decreased and TEM analyses showed a high amount of spherical particles compared to using $20 \mu \mathrm{l}$ seed addition and 0.1M CTAB even though L-LSPR peak positions were similar (fig. S1).

\subsection{Interplay of various tuning methods and TEM analyses}

The silver to gold ratio, acidity, CTAB concentration and seed addition tuning strategies were combined in an effort to obtain nanorods with similar L-LSPR peaks using various strategies. Synthesis combinations that led to nanoparticles with L-LSPR peaks around 700, 760, 784, 845 and $867 \mathrm{~nm}$ were characterised by TEM (fig. 3). Table 1 summarizes the parameters used for each synthesis (a-h) of the particles shown. The estimated final yield for each synthesis (except $d$-e) was based on the use of 
experimentally determined epsilon values obtained by Orendorff for nanoparticles with comparable L-LSPR peaks and aspect ratios [31]. For synthesis $d$ and e, the particles did not fall within these size dimensions. Thus, discrete dipole approximation calculations would need to be made to obtain a theoretical epsilon [37]. This was considered out of scope for this work. When comparing TEM images of synthesis $\mathrm{a}, \mathrm{b}$ and $\mathrm{c}$ (all with an L-LSPR $700 \mathrm{~nm}$ ) it becomes clear that varying the seed addition, $\mathrm{pH}$, silver to gold ratio variation and final CTAB concentration is crucial to obtain homogenous nanorods with a L-LSPR around $700 \mathrm{~nm}$. Indeed, only the combination used for the synthesis shown in fig. 3c led to the production of homogenous rods for this wavelength. This is probably due to a balance between a reduced growth kinetic caused by higher CTAB concentrations countered by a slightly lower redox potential of ascorbic acid at lower acidity and a reduction in directed anisotropic growth associated with lower silver concentrations. These counter balancing conditions may have caused the nanorods to be formed at just the right rate to avoid too many spherical by products. In the other synthesis (fig. 3a-b) the kinetics of the reaction may have been overly increased causing the formation of irregularities. The synthesis $\mathrm{f}-\mathrm{h}$ produced homogenous nanorods with L-LSPRs between 785 and $867 \mathrm{~nm}$. The yield of the particles synthesised in $\mathrm{f}$ was the highest $\left(0.35 \mathrm{nM} \cdot \mathrm{mL}^{-1}\right)$ which was $\sim 25 \%$ higher as synthesis $h$ (the next best yield) and synthesis c. Finally, in agreement with the observation derived from fig. $2 \mathrm{~d}$, it seems that good homogenous rods with an L-LSPR peak above $800 \mathrm{~nm}$ are best produced using $0.1 \mathrm{M} \mathrm{CTAB}$ at acidic conditions and can be fine-tuned by varying seed addition ( $\mathrm{g}$ and h). Table 2 shows the average length, width and aspect ratio of the nanorods synthesised using synthesis $f-h$, which were considered the most promising for coupling experiments in terms of high yield estimations, narrow UV-Vis spectra and homogeneous TEM images showing little impurities. All three synthesis produced nanorods with length and width variations of $\sim 10 \%$ and $\sim 13 \%$. The AR of the nanorods of synthesis $f$ were closest to the previously determined optimum AR of 3.3 for plasmonic biosensing [10]. Moreover, the quantum efficiency of CCD or CMOS camera is good at the L-LSPR of these particles $(785 \mathrm{~nm})$. Thus, the nanorods of synthesis $\mathrm{f}$ were used in all further experiments. The UV-Vis spectra of the nanospheres and nanorods used from hereon are shown in fig. S2. 


\subsection{Biosensor construction}

\subsubsection{Nanoparticle functionalization}

PEGylation of the nanoparticles was initially performed using a molar ratio of 1:3 0.46 kDa PEGacid: mPEG. This successfully stabilized the nanospheres but the nanorods aggregated. It is known that the exchange rate of CTAB for PEG chains is much lower than that of citrate capped particles making the PEGylation of CTAB stabilized nanorods capricious [38]. The molecular structure, length and charge of the PEG molecule can drastically influence the chemical stabilisation of the nanorods [39] [40]. Thus, attempts were made to stabilise the nanorods using additional PEG mixtures (fig. 4a). Only the $1 \mathrm{kDa}$ PEGamine SAM led to stable nanorods and was used from hereon. GPTMS derived glass slides were functionalised with nanorods using 1:4, 1:8 and 1:16 dilutions of the PEGylated nanorods in PBS. DFM images were taken with a fixed shutter time and gain (fig 4c). Up until the 1:8 dilution, inter-particle distances are small and aggregation can be seen. At 1:16 almost no large aggregation was observed and particles appeared red-green indicating inter-particle distance was sufficient. Thus, this dilution was used from hereon. Additionally, the PEGylated nanospheres were equally immobilised on the silica support using different dilutions to verify if these particles can produce sufficient Rayleigh scatter intensity to be detected with DFM (fig. S3). The figure clearly shows the NPs produce good scatter intensity and are easily detected by DFM. Moreover, at the last dilution (1:16) almost no large aggregation is observed and most particles appear blue indicating that the inter-particle distance is sufficient to prevent bulk plasmon coupling [41]. The immobilised nanorods were conjugated to BSADA. Conjugation success of immobilised nanorods to BSA-DA was determined through a fluorescence assay using anti-IgG-TRITC as a label (fig. $4 \mathrm{~b}$ ). The $\mathrm{S} / \mathrm{N}$ ratios for BSA-DA conjugated nanorods incubated with AntiDA-mAb and anti-IgG-TRITC was significantly different to the $\mathrm{S} / \mathrm{N}$ ratio obtained when bare or BSA-DA conjugated nanorods were directly incubated with anti-IgG-TRITC (Tukey post hoc; $p<0.0001$ ) confirming successful conjugation. 


\subsubsection{Domoic acid detection.}

The capability of two different methods to detect DA were investigated. With the first method, a plasmonic coupling experiment between NR-BSA-DA and AntiDA-NP was conducted. A large average blue shift $(55 \mathrm{~nm})$ was detected (fig $5 \mathrm{a}$ ) resulting from an overall blue shift of the L-LSPR peaks of individual nanoparticles (fig. 5b). This was unexpected since plasmon coupling generally causes a red shift. The blue shift may be accorded to repulsive polarisation of high-energy anti-symmetric field longitudinal plasmons of the NR-BSA-DA with redshifted plasmons from the BSA-DA-NP that bound in close proximity on the NR-BSA-DA as explained by the electromagnetic variant of the molecular orbital model [42], [43]. An alternative explanation is that nanosphere-nanorod interaction led to the formation of sphere-like aggregations with an average extinction spectrum that was blue shifted from the NR-BSA-DA spectra. In any case the observed high coupling effect is likely caused by cluster formation of multiple AntiDA-NP binding with single NR-BSA-DA particles [6]. However detailed high resolution TEM imaging and theoretical calculations may be needed to further demonstrate the physics behind this type of particle interaction. With the second method, only the pixels in the image are quantified and no spectral reconstruction is performed. Fig. $5 c$ shows the pixel count per spot for the coupling experiments of NR-BSA-DA with AntiDA-NP, NR-BSA-DA with NP-Aspec-mAb, NR-BSA-DA with AntiDA-NP and $10 \mu \mathrm{M}$ DA addition and, NR-BSA-DA with PEG-NPs. DFM images of the positive control (the coupling experiment between NR-DA-BSA and AntiDA-NP) and negative control (the coupling experiment between NR-DA-BSA and NP-PEG) show that particle aggregation was highly visible for the positive but absent for the negative control (fig. $5 \mathrm{~d}$ ). A paired two-way ANOVA on the pixel counts was significant for the row factor (before and after the coupling experiments; $p=0.0002$ ), column factor (type of NP conjugation; $p=0.02$ ) and interaction $(p=0.001)$. Sidak's multiplicity corrected post-hoc analyses comparing each cell mean with the other cell mean in that row showed that only for the NR-BSA-DA/AntiDA-NP interaction there was a significant increase in the pixel count per spot ( $p=0.0005$; fig. $5 c$ ). Finally, the signal disappeared if AntiDA-NP was mixed with DA prior to the reaction (multiplicity corrected Sidak's post hoc $p$ value $=0.39$ ) while the RSD for the inter spot 
variation was satisfactory $(<15 \%)$ which corresponds with the previously obtained optimal reproducibility of such nanoarrays for LSPR based detection [44]. Overall the study showed that the rapid qualitative detection of DA with this method is possible at a DA concentration of $10 \mu \mathrm{M}$. It is likely that the assay can even detect lower concentrations of DA since the chosen limit could be detected with good statistic certainty. However, this was not considered necessary since the chosen concentration is already at only $15 \%$ of the current maximum residue limit for DA in shellfish in the EU [45].

\subsection{Conclusion}

The interplay of seed addition, acidity, silver to gold ratio and CTAB concentration in the growth solution enabled the synthesis of homogeneous nanorods with various aspect ratios and resulted in the optimization of production protocols for nanorods over a $700-900 \mathrm{~nm}$ range. Spectroscopic and electron microscopic analyses showed that $12 \mu \mathrm{l} \mathrm{HCl}, 20 \mu \mathrm{l}$ seed addition, $0.05 \mathrm{M} \mathrm{CTAB}$ and a silver to gold ratio of 1:5 resulted in the synthesis of nanorods with an aspect ratio of 3.5. This is close to the optimal aspect ratio for plasmonic bio-sensing and the L-LSPR peak wavelength $(785 \mathrm{~nm})$ is well within the detection range of CCD or CMOS cameras. These nanorods were then immobilised on a silica support, functionalised with a domoic acid protein conjugate and used for plasmonic coupling experiments with domoic acid antibody conjugated nanospheres. The plasmon coupling of these particles resulted in a large blue-shift $(55 \mathrm{~nm})$ of the L-LSPR peak in the average extinction spectrum. Additionally, in a second system only pixel counts were quantified from the images. This method showed the specific binding of the AntiDA-NPs to the NR-BSA-DA could be statistically distinguished while no statistically significantly aspecific binding of nanospheres functionalised with aspecific antibody was observed. Moreover, pixel counts per spot returned to initial values if domoic acid was added to the immunoassay at $10 \mu \mathrm{M}$, a concentration $>6$ fold below the EU MRL [45]. Thus, this method merits further development and evaluation as there is clear potential as a cost-efficient, simple and rapid ( 30 minutes which may be reduced with further optimisation) high throughput screening method for the detection of trace contaminants. Such alternative tools and methodology as 
precursors for the advancement of sensor techniques for chemical contaminant and toxin detection using immunological approaches are continuously required. Although the method is currently qualitative, it is expected the method can be used for quantitative detection of domoic acid as well because of the large difference in the amount of pixels per spot between the tested DA concentration and the blank. However, this would need to be verified with a detailed quantitative study.

\section{Acknowledgements}

This project has received funding from the European Union's Horizon 2020 research and innovation program under the Marie Sklodowska-Curie Grant Agreement No 720325.

\section{References}

[1] J. N. Anker, W. P. Hall, O. Lyandres, N. C. Shah, J. Zhao, and R. P. Van Duyne, "Biosensing with plasmonic nanosensors," Nat. Mater., vol. 7, no. 6, pp. 442-453, 2008, doi: $10.1038 /$ nmat2162.

[2] N. Li, P. Zhao, and D. Astruc, "Anisotropic gold nanoparticles: Synthesis, properties, applications, and toxicity," Angew. Chemie - Int. Ed., vol. 53, no. 7, pp. 1756-1789, 2014, doi: 10.1002/anie.201300441.

[3] P. L. Truong, C. Cao, S. Park, M. Kim, and S. J. Sim, “A new method for non-labeling attomolar detection of diseases based on an individual gold nanorod immunosensor," Lab Chip, vol. 11, no. 15 , pp. 2591-2597, 2011, doi: 10.1039/c1lc20085b.

[4] S. Unser, I. Bruzas, J. He, and L. Sagle, "Localized Surface Plasmon Resonance Biosensing: Current Challenges and Approaches," Sensors (Basel)., vol. 15, no. 7, pp. 15684-15716, Jul. 2015, doi: 10.3390/s150715684.

[5] E. Martinsson, B. Sepulveda, P. Chen, A. Elfwing, B. Liedberg, and D. Aili, "Optimizing the Refractive Index Sensitivity of Plasmonically Coupled Gold Nanoparticles," Plasmonics, vol. 9, no. 4, pp. 773-780, 2014, doi: 10.1007/s11468-013-9659-y.

[6] K. Su, Q. Wei, and X. Zhang, "Interparticle Coupling Effects on Plasmon Resonances of Nanogold Particles," Nano Lett., vol. 3, no. 8, pp. 1087-1090, 2003, doi: 10.1021/nl034197f.

[7] W. P. Hall, S. N. Ngatia, and R. P. Van Duyne, "LSPR Biosensor Signal Enhancement Using Page $\mid 16$ 
Nanoparticle - Antibody Conjugates," Jounal Phys. Chem. C, vol. 115, pp. 1410-1414, 2011, doi: dx.doi.org/10.1021/jp106912p.

[8] L. P. F. Peixoto, J. F. L. Santos, and G. F. S. Andrade, "Plasmonic nanobiosensor based on Au nanorods with improved sensitivity: A comparative study for two different configurations," Anal. Chim. Acta, vol. 1084, pp. 71-77, 2019, doi: https://doi.org/10.1016/j.aca.2019.07.032.

[9] C.-D. Chen, S.-F. Cheng, L.-K. Chau, and C. R. C. Wang, "Sensing capability of the localized surface plasmon resonance of gold nanorods," Biosens. Bioelectron., vol. 22, no. 6, pp. $926-$ 932, 2007, doi: 10.1016/j.bios.2006.03.021.

[10] J. Becker, A. Trügler, A. Jakab, U. Hohenester, and C. Sönnichsen, "The optimal aspect ratio of gold nanorods for plasmonic bio-sensing," Plasmonics, vol. 5, no. 2, pp. 161-167, 2010, doi: 10.1007/s11468-010-9130-2.

[11] B. Nikoobakht and M. A. El-Sayed, "Preparation and growth mechanism of gold nanorods (NRs) using seed-mediated growth method," Chem. Mater., vol. 15, no. 10, pp. 1957-1962, 2003, doi: 10.1021/cm020732I.

[12] R. M. Pallares, X. Su, S. H. Lim, and N. T. K. Thanh, "Fine-tuning of gold nanorod dimensions and plasmonic properties using the Hofmeister effects," J. Mater. Chem. C, vol. 4, no. 1, pp. 53-61, 2016, doi: 10.1039/C5TC02426A.

[13] C. J. M. Nikhil R. Jana, Latha Gearheart, "Seed-Mediated Growth Approach for ShapeControlled Synthesis of Spheroidal and Rod-like Gold Nanoparticles Using a Surfactant Template," Adv. Mater., no. 18, pp. 1389-1393, 2001.

[14] J. Pérez-Juste, M. A. Correa-Duarte, and L. M. Liz-Marzán, "Silica gels with tailored, gold nanorod-driven optical functionalities," Appl. Surf. Sci., vol. 226, no. 1-3 SPEC. ISS., pp. 137143, 2004, doi: 10.1016/j.apsusc.2003.11.013.

[15] J. Cheng, L. Ge, B. Xiong, and Y. He, "Investigation of pH Effect on Gold Nanorod Synthesis," J. Chinese Chem. Soc., vol. 58, no. 6, pp. 822-827, 2011, doi: 10.1002/jccs.201190128.

[16] C. Wang, T. Wang, Z. Ma, and Z. Su, "PH-tuned synthesis of gold nanostructures from gold nanorods with different aspect ratios," Nanotechnology, vol. 16, no. 11, pp. 2555-2560, 2005, doi: 10.1088/0957-4484/16/11/015.

[17] C. Bullen, P. Zijlstra, E. Bakker, M. Gu, and C. Raston, "Chemical kinetics of gold nanorod growth in aqueous CTAB solutions," Cryst. Growth Des., vol. 11, no. 8, pp. 3375-3380, 2011, doi: $10.1021 / \operatorname{cg} 101636 r$.

Page | 17 
[18] J. Pérez-Juste, I. Pastoriza-Santos, L. M. Liz-Marzán, and P. Mulvaney, “Gold nanorods: Synthesis, characterization and applications," Coord. Chem. Rev., vol. 249, no. 17-18 SPEC. ISS., pp. 1870-1901, 2005, doi: 10.1016/j.ccr.2005.01.030.

[19] M. Reza Hormozi-Nezhad, H. Robatjazi, and M. Jalali-Heravi, "Thorough tuning of the aspect ratio of gold nanorods using response surface methodology," Anal. Chim. Acta, vol. 779, pp. 14-21, 2013, doi: https://doi.org/10.1016/j.aca.2013.03.056.

[20] L. Li, B. Liu, and Z. Chen, "Colorimetric and dark-field microscopic determination of cadmium(II) using unmodified gold nanoparticles and based on the formation of glutathionecadmium(II) complexes," Microchim. Acta, vol. 186, no. 1, p. 37, 2018, doi: 10.1007/s00604018-3166-y.

[21] C.-Y. Poon, L. Wei, Y. Xu, B. Chen, L. Xiao, and H.-W. Li, "Quantification of Cancer Biomarkers in Serum Using Scattering-Based Quantitative Single Particle Intensity Measurement with a Dark-Field Microscope," Anal. Chem., vol. 88, no. 17, pp. 8849-8856, Sep. 2016, doi: 10.1021/acs.analchem.6b02429.

[22] A. Campbell, D. Hudson, C. Mcleod, C. Nicholls, and A. Pointon, "The 2012-13 paralytic shellfish toxin event in Tasmania associated with the dinoflagellate alga Alexandrium tamarense," FRDC Proj. 2012/060, SafeFish, Adelaide., vol. 060, 2013.

[23] D. Anderson, "HABs in a changing world: a perspective on harmful algal blooms, their impacts, and research and management in a dynamic era of climactic and environmental change," Harmful Algae, vol. 2010, no. 2012, pp. 3-17, 2012.

[24] J. B. M. B.D. Gessner, "Seafood and Freshwater Toxins: Pharmacology, Physiology, and Detection," J. Aquat. Food Prod. Technol., 2000, doi: 10.1080/10498850.2015.1024964.

[25] FAO, "The State of World Fisheries and Aquaculture 2018 - Meeting the sustainable development goals.," Rome, 2018. [Online]. Available: http://www.fao.org/3/i9540en/I9540EN.pdf?segid=b273defb-c3b2-4146-9f57b1db87877cfb.

[26] J. L. D. Nelis et al., "The benefits of carbon black, gold and magnetic nanomaterials for pointof-harvest electrochemical quantification of domoic acid," Microchim. Acta, vol. 187, no. 3, 2020, doi: 10.1007/s00604-020-4150-x.

[27] B. J. Yakes, J. Buijs, C. T. Elliott, and K. Campbell, "Surface plasmon resonance biosensing: Approaches for screening and characterising antibodies for food diagnostics," Talanta, vol. 
156-157, pp. 55-63, 2016, doi: 10.1016/j.talanta.2016.05.008.

[28] J. Turkevich, "Colloidal gold. Part I," Gold Bull., vol. 18, no. 4, pp. 125-131, 1985, doi: 10.1007/BF03214694.

[29] C. Mcvey, N. Logan, N. T. K. Thanh, C. Elliott, and C. Cao, "Unusual switchable peroxidasemimicking nanozyme for the deter- mination of proteolytic biomarker," Nano Res., vol. 12, no. 1, pp. 1-8, 2019, doi: 10.1007/s12274-018-2241-3.

[30] W. Haiss, N. T. K. Thanh, J. Aveyard, and D. G. Fernig, "Determination of Size and Concentration of Gold Nanoparticles from UV," Anal. Chem., vol. 79, no. October, pp. 42154221, 2007, doi: 10.1021/ac0702084.

[31] C. J. Orendorff and C. J. Murphy, "Quantitation of metal content in the silver-assisted growth of gold nanorods," J. Phys. Chem. B, vol. 110, no. 9, pp. 3990-3994, 2006, doi: 10.1021/jp0570972.

[32] I. Mannelli and M.-P. Marco, "Recent advances in analytical and bioanalysis applications of noble metal nanorods," Anal. Bioanal. Chem., vol. 398, no. 6, pp. 2451-2469, 2010, doi: 10.1007/s00216-010-3937-8.

[33] N. Tort, J.-P. Salvador, and M.-P. Marco, "Multimodal plasmonic biosensing nanostructures prepared by DNA-directed immobilization of multifunctional DNA-gold nanoparticles," Biosens. Bioelectron., vol. 90, pp. 13-22, 2017, doi: https://doi.org/10.1016/j.bios.2016.11.022.

[34] F. Fernández, F. Sánchez-Baeza, and M. P. Marco, "Nanogold probe enhanced Surface Plasmon Resonance immunosensor for improved detection of antibiotic residues," Biosens. Bioelectron., vol. 34, no. 1, pp. 151-158, 2012, doi: 10.1016/j.bios.2012.01.036.

[35] Y. H. Tan, M. Liu, B. Nolting, J. G. Go, J. Gervay-Hague, and G. Y. Liu, "A nanoengineering approach for investigation and regulation of protein immobilization," ACS Nano, vol. 2, no. 11, pp. 2374-2384, 2008, doi: 10.1021/nn800508f.

[36] P. Zamora-Perez, D. Tsoutsi, R. Xu, and P. Rivera-Gil, "Hyperspectral-enhanced dark field microscopy for single and collective nanoparticle characterization in biological environments," Materials (Basel)., vol. 11, no. 2, p. 11020243, 2018, doi: $10.3390 /$ ma11020243.

[37] R. D. Near, S. C. Hayden, R. E. Hunter, D. Thackston, and M. A. El-Sayed, "Rapid and efficient prediction of optical extinction coefficients for gold nanospheres and gold nanorods," J. Phys. Page | 19 
Chem. C, vol. 117, no. 45, pp. 23950-23955, 2013, doi: 10.1021/jp4082596.

[38] X. Xia et al., "Quantifying the coverage density of poly(ethylene glycol) chains on the surface of gold nanostructures," ACS Nano, vol. 6, no. 1, pp. 512-522, 2012, doi: 10.1021/nn2038516.

[39] F. Schulz, W. Friedrich, K. Hoppe, T. Vossmeyer, H. Weller, and H. Lange, "Effective PEGylation of gold nanorods," Nanoscale, vol. 8, no. 13, pp. 7296-7308, 2016, doi:

10.1039/C6NR00607H.

[40] Y. Liu, M. K. Shipton, J. Ryan, E. D. Kaufman, S. Franzen, and D. L. Feldheim, “Synthesis, stability, and cellular internalization of gold nanoparticles containing mixed peptidepoly(ethylene glycol) monolayers," Anal. Chem., vol. 79, no. 6, pp. 2221-2229, 2007, doi: 10.1021/ac061578f.

[41] M. P. Kreuzer, R. Quidant, J. P. Salvador, M. P. Marco, and G. Badenes, "Colloidal-based localized surface plasmon resonance (LSPR) biosensor for the quantitative determination of stanozolol," Anal. Bioanal. Chem., vol. 391, no. 5, pp. 1813-1820, 2008, doi: 10.1007/s00216008-2022-z.

[42] E. Prodan, C. Radloff, N. J. Halas, and P. Nordlander, "A Hybridization Model for the Plasmon Response of Complex Nanostructures," Science (80-. )., vol. 302, no. 5644, pp. 419-422, 2003, doi: 10.1126/science.1089171.

[43] P. Nordlander, C. Oubre, E. Prodan, K. Li, and M. I. Stockman, "Plasmon Hybridization in Nanoparticle Dimers," Nano Lett., vol. 4, no. 5, pp. 899-903, 2004, doi: 10.1021/nl049681c.

[44] M. P. Kreuzer, R. Quidant, J.-P. Salvador, M.-P. Marco, and G. Badenes, “Colloidal-based localized surface plasmon resonance (LSPR) biosensor for the quantitative determination of stanozolol," Anal. Bioanal. Chem., vol. 391, no. 5, pp. 1813-1820, 2008, doi: 10.1007/s00216008-2022-z.

[45] A. S. Tsagkaris et al., "Critical assessment of recent trends related to screening and confirmatory analytical methods for selected food contaminants and allergens," TrAC Trends Anal. Chem., vol. 121, p. 115688, 2019, doi: https://doi.org/10.1016/j.trac.2019.115688. 


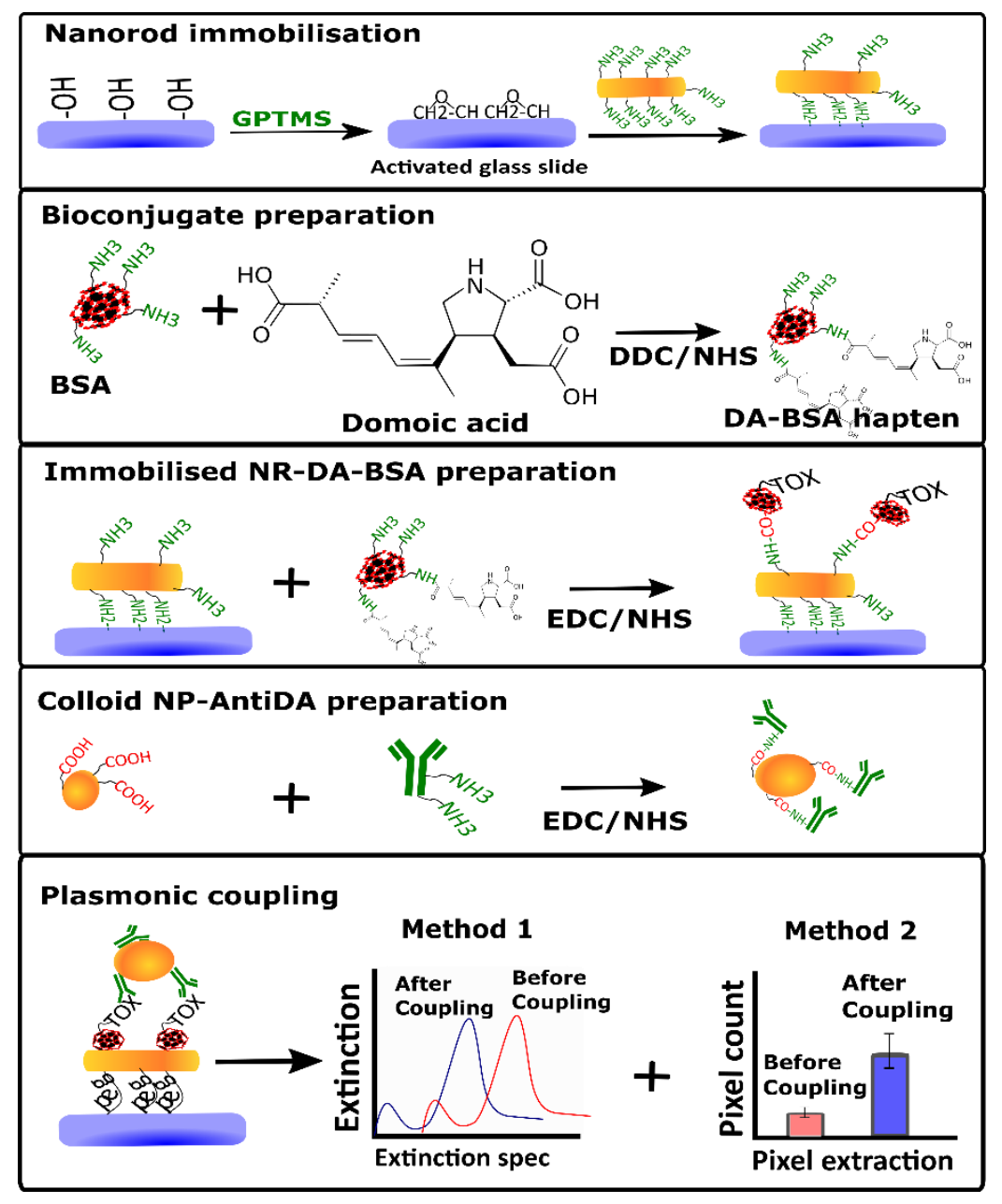

Fig. 1. Scheme depicting the development of the biosensor, the immunoassay and the two different systems to analyse the plasmonic coupling data. 

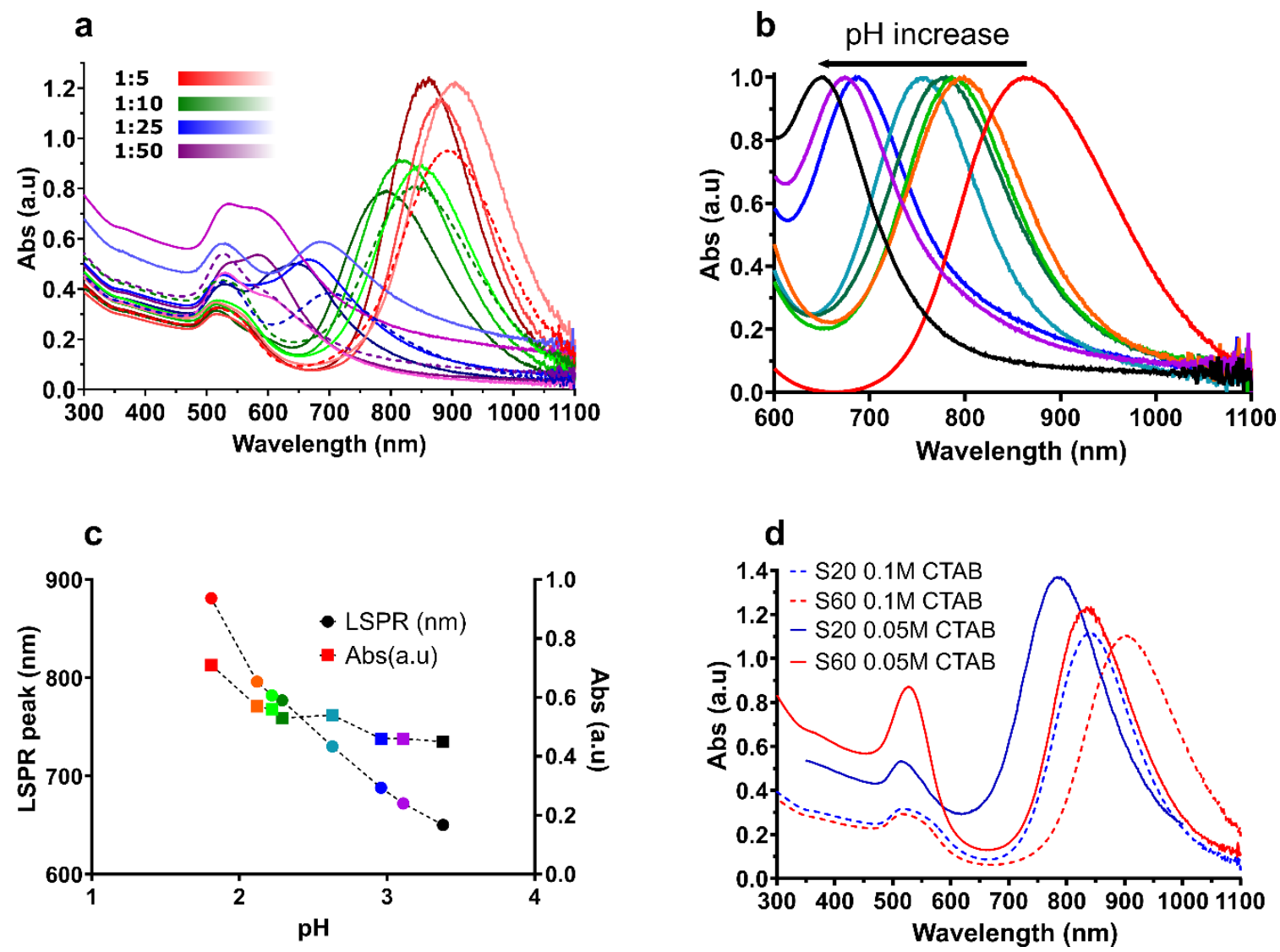

Fig. 2. UV-Vis spectra of nanorods using various tuning approaches. a) Spectra of nanorods synthesised using 1:5 (red), 1:10 (green), 1:25 (blue) and 1:50 (purple) molar silver to gold ratios and 0.05M CTAB under acidic conditions ( $12 \mu \mathrm{l}$ concentrated $\mathrm{HCL}$ added to growth solution). Seed addition was varied

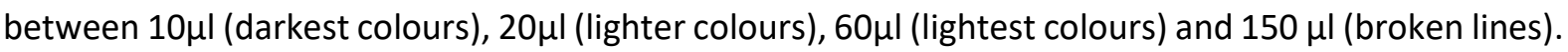
b) Normalised spectra of nanorods synthesized at varying $\mathrm{pH}$. Colours (from red to black) stand for growth reactions with decreasing $\mathrm{HCl}(37 \%)$ addition with $12 \mu \mathrm{l}$ (red), $8 \mu$ (orange), $6 \mu$ (light green), $4 \mu \mathrm{l}$ (dark green), $2 \mu \mathrm{l}$ (light blue), $0 \mu \mathrm{l}$ (blue) $\mathrm{HCL}$ addition or $2 \mu \mathrm{l}$ (magenta) and $4 \mu \mathrm{l}$ (black) $\mathrm{NaOH}$ addition. c) Wavelength (circles; left y-axes) and absorbance (squares; right $y$-axes) values at the LLSPR peak of the particles synthesised at varying $\mathrm{pH}$ as shown in (b). The $\mathrm{pH}$ values indicate final $\mathrm{pH}$ concentration after completing the reaction. The colour of each point corresponds with the VIS-NIR spectra shown in (b). d) Spectra of nanorods synthesised using $20 \mu \mathrm{l}$ (blue) or $60 \mu \mathrm{l}$ (red) seeds in a growth pot containing $0.05 \mathrm{M}$ (solid) or $0.1 \mathrm{M}$ (dashed) CTAB concentration with $12 \mu$ ladded $\mathrm{HCl}(37 \%)$ and a 1:5 silver to gold ratio. 


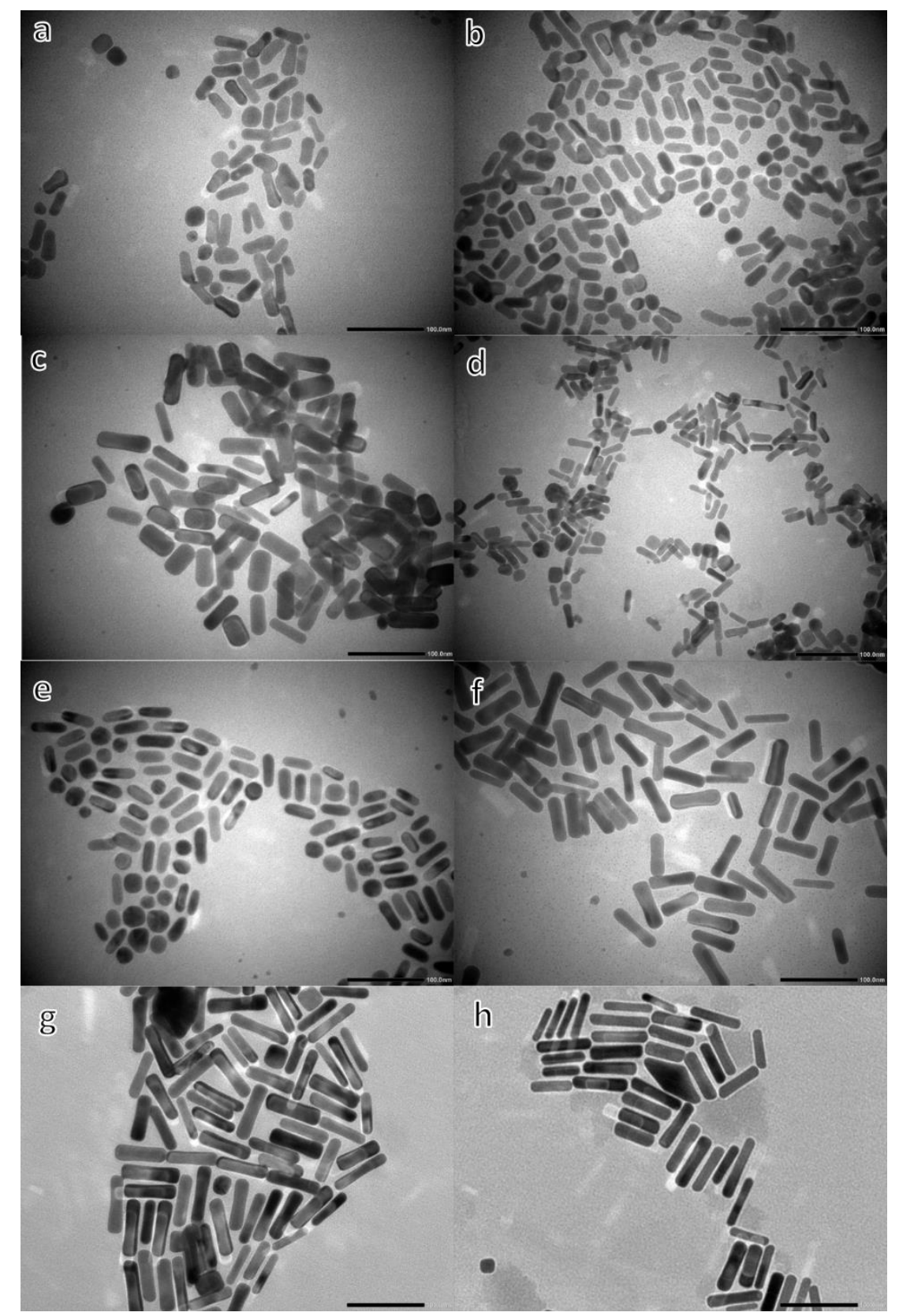

Fig. 3. TEM images of various nanorod synthesis. a-c) Images of nanorods with a L-LSPR of $\sim 700 \mathrm{~nm}$ (708, 699 and $695 \mathrm{~nm}$ respectively). d-e nanorods with an L-LSPR of $\sim 760 \mathrm{~nm}(769,762)$. f) Nanorods with an L-LSPR of $784 \mathrm{~nm}$. g-h) Nanorods with an L-LSPR at 845 and $867 \mathrm{~nm}$ respectively. All images of nanorods were taken at $100 \mathrm{~K}$ magnification. Scale bars indicate $100 \mathrm{~nm}$ distance. Details of each nanorod synthesis are given in table 1. 
a

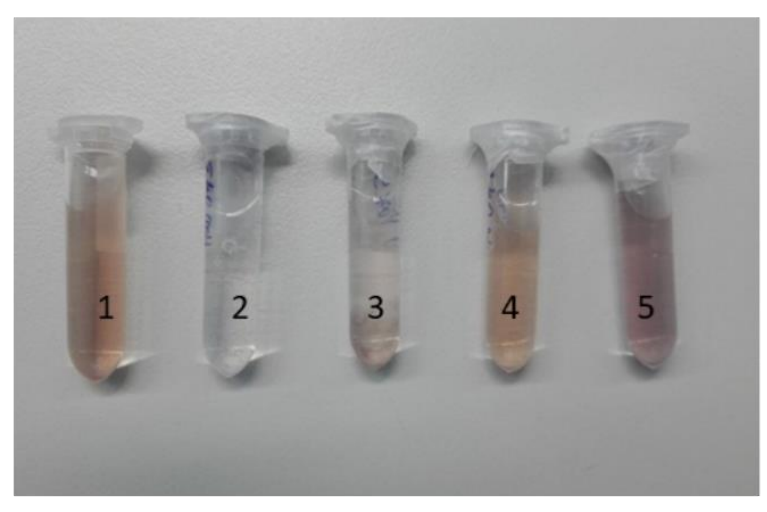

C

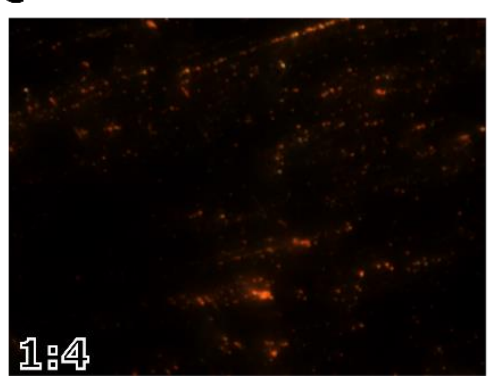

b

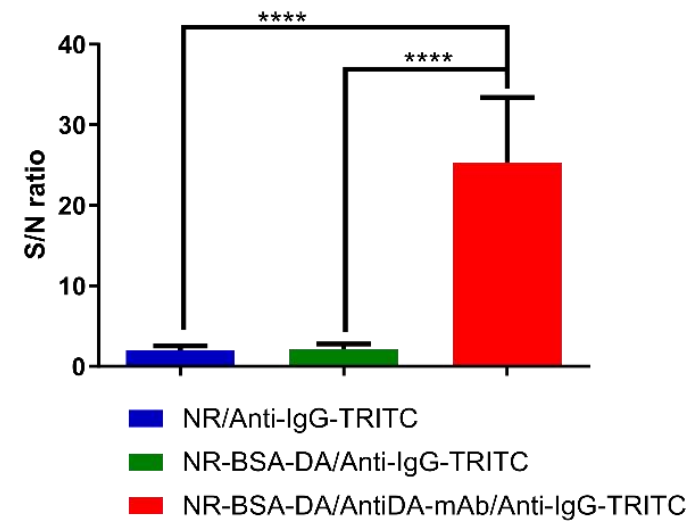

Fig 4. Biosensor development. a) NR stabilization test using four PEG combinations. From left to right the following combinations were left to react overnight with 1) no added PEG, (nanorods are CTAB stabilised). 2) 5 kDa PEGacid. 3) 5 kDa PEGacid mixed with mPEG in a 1:3 ratio. 4) 1 kDa PEGamine. 5) $1 \mathrm{kDa}$ PEGamine and mPEG in a 1:3 ratio. b) Signal over noise (S/N) ratio of anti-IgG-TRITC fluorescence response to bare immobilised nanorods (NR/anti-IgG-TRITC), immobilised nanorods functionalised with BSA-DA (NR-BSA-DA/anti-IgG-TRITC) and BSA-DA functionalised immobilised nanorods functionalised after incubation with AntiDA-mAb (NR-BSA-DA/AntiDA-mAb/anti-IgG-TRITC). One-way ANOVA was highly significant $(p<0.0001)$. Significant Tukey post hoc tests are indicated $(* * * *=p<0.0001) . c)$ DFM images $(40 x)$ of immobilised nanorods at various dilutions in PBS. A fixed shutter time (63 ms) and gain (8\%) was used in all conditions. 
a

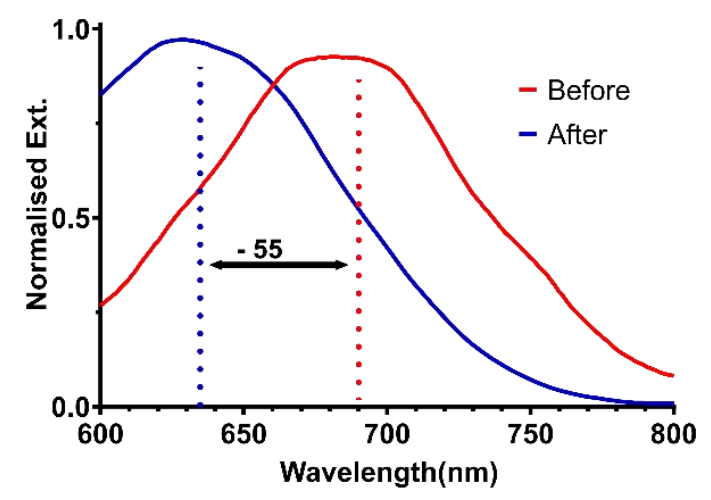

C

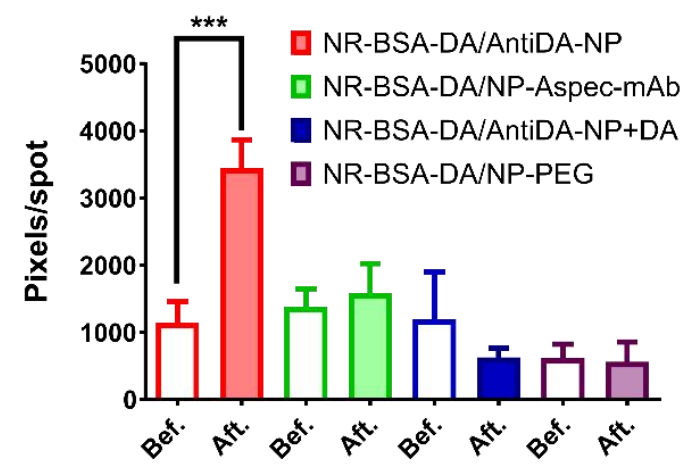

b

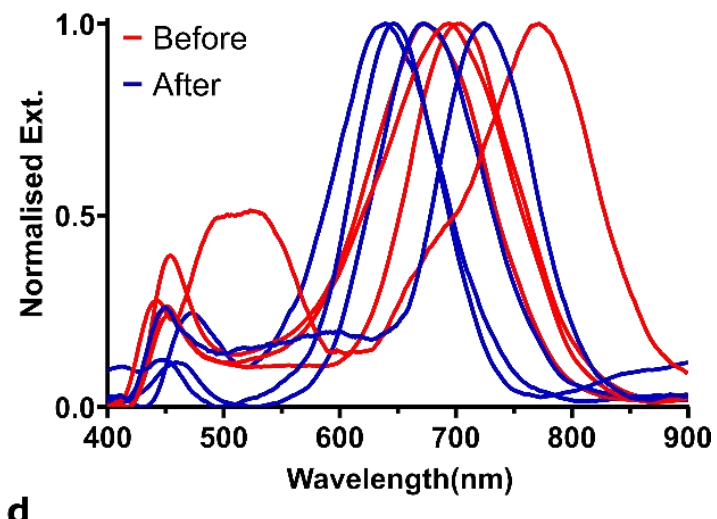

d
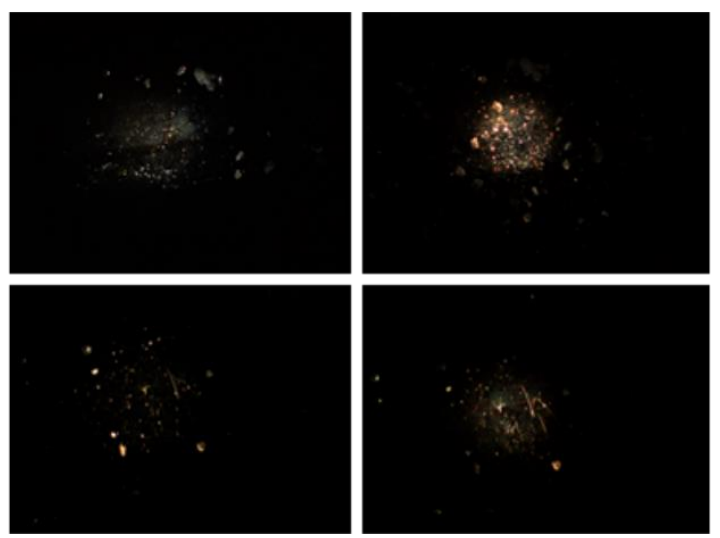

Fig. 5. Plasmonic coupling based domoic acid detection via two methods. a) Average LSPR spectra constructed from individual immobilised NR-DA-BSA particles present in the same spot before (red) and after (blue) reaction with AntiDA-mAb. b) Extinction spectra of individual particles used for the average spectra represented in (a). c) The amount of pixels per spot extracted from hyperspectral images from spots of immobilised NR-BSA-DA after (closed bars) and before (open bars) reacting with AntiDA-NP (red), NP-Aspec-mAb (green), AntiDA-NP mixed with $10 \mu \mathrm{m}$ DA (blue) and NP-PEG (violet) $(n=3)$. The significant difference in pixel/spot count before and after the immunoreaction is indicated (Sidak's multiplicity corrected $p=0.005) d$ ) A typical DFM image ( $4 x$ objective) of the spots before (left) and after (right) reaction with AntiDA-NP (top) and NP-PEG (bottom). 
Table 1. Specifications of synthesis used for nanoparticles shown in fig. 3. Added $\mathrm{HCl}(37 \%)$ and seed (S) addition to growth solution is given in $\mu$. CTAB concentration ( 0.05 or $0.1 \mathrm{M})$ in growth solution and gold to silver ratio (1:5 or 1:10) is also indicated. n.a is not available.

\begin{tabular}{|c|c|c|c|c|}
\hline $\begin{array}{l}\text { Nanoparticles in } \\
\text { fig. } 3\end{array}$ & Synthesis & $\begin{array}{l}\text { LSPR } \max \\
(n m)\end{array}$ & $\begin{array}{l}\text { Yield } \\
(n M / m L)\end{array}$ & Description of shape \\
\hline$a$ & $\begin{array}{l}2 \mathrm{HCl}, 40 \mathrm{~S}, 0.05 \mathrm{M} \\
\text { CTAB, 1:5 }\end{array}$ & 708 & 0.18 & Irregular forms \\
\hline$b$ & $\begin{array}{l}0 \mathrm{HCl}, 60 \mathrm{~S}, 0.05 \mathrm{M} \\
\text { CTAB, 1:5 }\end{array}$ & 699 & 0.16 & Irregular forms \\
\hline$c$ & $\begin{array}{l}8 \mathrm{HCl}, 20 \mathrm{~S}, 0.1 \mathrm{MCTAB}, \\
1: 10\end{array}$ & 695 & 0.25 & More regular rods \\
\hline$d$ & $\begin{array}{l}\text { No } \mathrm{HCl}, 60 \mathrm{~S}, 0.1 \mathrm{M} \\
\text { CTAB, 1:5 }\end{array}$ & 769 & n.a & $\begin{array}{l}\text { Thin rods with minor } \\
\text { impurities }\end{array}$ \\
\hline$e$ & $\begin{array}{l}6 \mathrm{HCl}, 60 \mathrm{~S}, 0.05 \mathrm{CTAB}, \\
1: 5\end{array}$ & 762 & n.a & $\begin{array}{l}\text { Thicker rods with } \\
\text { impurities }\end{array}$ \\
\hline$f$ & $\begin{array}{l}12 \mathrm{HCl}, 20 \mathrm{~S}, 0.05 \mathrm{M} \\
\text { CTAB, 1:5 }\end{array}$ & 785 & 0.35 & Homogenous rods \\
\hline$g$ & $\begin{array}{l}12 \mathrm{HCL}, 20 \mathrm{~S}, 0.1 \mathrm{M} \\
\text { CTAB, 1:5 }\end{array}$ & 845 & 0.26 & Homogenous rods \\
\hline$h$ & $\begin{array}{l}12 \mathrm{HCL}, 30 \mathrm{~S}, 0.1 \mathrm{M} \\
\text { CTAB, 1:5 }\end{array}$ & 867 & 0.28 & Homogenous rods \\
\hline
\end{tabular}


Table 2: Size measurement of the particles displayed in fig. 3. A random set $(n=75)$ of particles using the synthesis f-h were measured using Image J.

$\begin{array}{llll}\text { Synthesis } & g & h & f \\ \text { Length }(\mathrm{nm}) & 63.2 \pm 6.8( \pm 10.7 \%) & 63.6 \pm 5.7( \pm 9.0 \%) & 54.9 \pm 5.3( \pm 9.6 \%) \\ \text { Width }(\mathrm{nm}) & 13.4 \pm 1.8( \pm 13.4 \%) & 12.8 \pm 1.6(12.5 \%) & 15.7 \pm 2.1( \pm 13.3 \%) \\ \text { Aspect ratio } & 4.7 & 5.0 & 3.5 \\ \text { L-LSPR }_{\max }(\mathrm{nm}) & 845 & 867 & 785\end{array}$

